PTH-132 HIGH RATES OF CLINICAL RESPONSE ARE MAINTAINED AFTER SWITCHING FROM ORIGINATOR TO BIOSIMILAR INFLIXIMAB

Rebecca Haggarty, Katy Waddell*, Jennifer Veryan, Emma Nowell, John Paul Seenan, Jonathan Macdonald. Queen Elizabeth University Hospital, Glasgow, UK

\subsection{6/gutjnl-2019-BSGAbstracts.191}

Introduction Biosimilar infliximab (BI) has been widely adopted in to clinical practice since launch in 2015. Switching patients with Inflammatory Bowel Disease (IBD) from originator infliximab (OI) to BI was endorsed by the BSG and ECCO despite a lack of data on the long-term implications of this strategy. Infliximab discontinuation (ID) occurs in $20-40 \%$ patients per annum. It is unclear whether switching to BI affects ID rates. This study assessed long-term outcomes after a managed BI switch programme.

Methods Individuals with Crohn's disease (CD) and Ulcerative Colitis (UC) who were changed from OI to BI in a switch programme in Dec 2016 were reviewed via electronic patient records. Demographics and pre-switch disease characteristics were recorded. Pre-switch medications and laboratory data were collected. Changes to BI dose/frequency at switch and during follow-up were recorded along with other changes to IBD medications. Post-switch durability of clinical response was evaluated. Rates of IBD related steroid use, hospitalisation and surgery were gathered. Incidence of adverse events was determined. Potential pre-switch indicators of future ID were explored.

Results 76 individuals considered to be clinically responding to OI were entered in to the BI switch programme. 2 patients had OI stopped prior to 1st dose BI based on clinical assessment and Therapeutic Drug Monitoring (TDM) results. 74 people were switched from OI to BI.

Abstract PTH-132 Table 1
\begin{tabular}{|c|c|}
\hline Characteristic & Number \\
\hline Median age & 42 (range 21-71) \\
\hline Male & 50 \\
\hline Female & 24 \\
\hline Smoker & 9 \\
\hline CD & 58 \\
\hline UC & 15 \\
\hline IBD unclassified & 1 \\
\hline Disease duration & \\
$<5$ years & 25 \\
$>5$ years & 49 \\
$>10$ years & 19 \\
$>20$ years & 10 \\
\hline
\end{tabular}

At time of switch 38 (51\%) were prescribed an immunomodulator. $52 / 74(70 \%)$ were on $5 \mathrm{mg} / \mathrm{kg} 8$ weekly treatment, the rest on higher doses. $48 / 74$ had a pre-switch FC, 32 (67\%) $\mathrm{FC}<250$. 61/70 (87\%), 69/73 (95\%) and 65/73 (89\%) had normal CRP, albumin and haemoglobin respectively $<3$ months prior to switch.

2 years post switch, 54/74 (73\%) remained on BI with sustained clinical response. ID was observed in 20 (27\%); 11 $(55 \%)$ now on another biologic, 8 (40\%) biologic free. 33/54 remaining on BI had treatment escalation at time of or subsequent to switch.
There was 1 non-treatment/IBD related death. 1 infusion reaction adverse event was observed. There were 6 IBD related surgeries, 4 unplanned hospital admissions and 12 required steroids for IBD flare. Evaluation of data identified no pre-switch factors associated with ID.

Conclusions Over 2 years of follow-up clinical response was maintained in the majority of individuals following BI switch. Rates of ID were lower than historically reported in nonswitched patient cohorts. BI switching appears to be a safe and effective intervention.

\section{PTH-133 THERAPEUTICDRUG MONITORING SUPPORTS CLINICAL DECISION MAKING WHEN EMPLOYED BEFORE AND AFTER BIOSIMILAR INFLIXIMAB SWITCHING}

Katy Waddell*, Rebecca Haggarty, Jennifer Veryan, Emma Nowell, John Paul Seenan, Jonathan Macdonald. Queen Elizabeth University Hospital, Glasgow, UK

\subsection{6/gutjnl-2019-BSGAbstracts.192}

Introduction Therapeutic drug and antibody monitoring (TDM) of infliximab (IFX) is used with increasing regularity as a tool to optimize outcomes in inflammatory bowel disease (IBD). Trough levels (TL) of $2-8 \mathrm{mcg} / \mathrm{ml}$ are recommended during maintenance IFX treatment. The introduction of biosimilar infliximab (BI) in 2015 lead to widespread switching of patients from originator infliximab (OI) to BI. The value of TDM when switching to BI has not been defined. This study aimed to assess the impact of TDM testing before and after a managed switch to BI.

Methods Individuals with IBD treated with OI and demonstrating a satisfactory response to treatment, were entered in to BI switch programme in Dec 2016. Pre-switch information was provided to patients, virtual or face to face clinical assessment was undertaken and it was recommended that all patients had pre-switch TDM performed. After switching patients returned to routine clinical care. Further TDM was performed at clinician discretion with recommendation to follow published TDM testing guidance. ${ }^{1}$ Virtual review of all patients was undertaken 2 years post-switch. Demographics, pre and post switch TDM data, OI and BI dosing regimens and other IBD related medications were recorded along with clinical outcome data. Comparative analysis of pre-switch and most contemporary TDM results was performed.

Results 76 individuals considered to be clinically responding to OI were entered in to the BI switch programme. 70/76 (92\%) had TDM at $<3$ months pre-switch. OI was discontinued prior to switch in 2 patients. 74 people were switched from OI to BI. $52 / 74(70 \%)$ were on $5 \mathrm{mg} / \mathrm{kg} 8$ weekly OI preswitch, 38 (51\%) were on immunomodulators.

Of 69/74 with pre-switch TDM, 32/69 (46\%) had subtherapeutic TLs $(<2 \mathrm{mcg} / \mathrm{ml})$. Median pre-switch TL was $2.2 \mathrm{mcg} /$ $\mathrm{ml}$ (IQR $1.1-3.4 \mathrm{mcg} / \mathrm{ml}$ ). Pre-switch TL review lead to $37 / 74$ $(50 \%)$ receiving an increased dose of $\mathrm{BI}$ at switch. In total $47 / 74$ had $\geq 1$ dose escalation at the time of or subsequent to switch.

$58 / 74(78 \%)$ had TDM testing in the 2 years after switch (median no. tests 2; range $1-5$ ) at which point 54/74 (73\%) remained on BI with sustained clinical response. 49 out of 54 still on BI had both pre and post switch TDM with results demonstrating a statistically significant increase in mean TLs ( 2.1 vs $6.3 \mathrm{mcg} / \mathrm{ml} ; \mathrm{p}<0.001)$; only $6 \%$ had persisting subtherapeutic TLs. 
Conclusions High rates of sustained clinical response were observed to occur following a BI switch supported by the use of pre and post switch TDM. TDM dose escalation resulted in a statistically significant increase in TLs, this may account for the rates of continued clinical response

\section{REFERENCE}

1. Papamichael K. Frontline Gastroenterol. 2016 Oct;7(4):289-300.

\section{PTH-134 EXPERIENCES OF USING VEDOLIZUMAB IN THE TREATMENT OF INFLAMMATORY BOWEL DISEASE IN THE EAST MIDLANDS}

\begin{abstract}
$1,2,3 J R$ White ${ }^{*},{ }^{3} S$ Din, ${ }^{4} R$ Ingram, ${ }^{4} S$ Foley, ${ }^{5} \mathrm{M}$ Alam, ${ }^{5} \mathrm{R}$ Robinson, ${ }^{1} \mathrm{R}$ Francis, ${ }^{1} \mathrm{E}$ Tucker, ${ }^{6} \mathrm{M}$ Jalal, ${ }^{6} \mathrm{D}$ Elphick, ${ }^{7} \mathrm{E}$ Atallah, ${ }^{7} \mathrm{~A}$ Norman, ${ }^{8} \mathrm{M}$ Amin, ${ }^{8} \mathrm{~A}$ Sajjad, ${ }^{9} \mathrm{~N}$ Heggs, ${ }^{9} \mathrm{~S}$ Meadowcroft, ${ }^{1,2} \mathrm{GW}$ Moran. ${ }^{1}$ Nottingham Digestive Diseases Centre, University of Nottingham, UK; ${ }^{2}$ NIHR BRC at Nottingham University Hospitals and University of Nottingham, UK; ${ }^{3}$ Royal Derby Hospital, UK; ${ }^{4}$ Kings Mill Hospital, Mansfield, UK; ${ }^{5}$ Leicester General Hospital, UK; ${ }^{6}$ Chesterfield Royal Hospital, UK; ${ }^{7}$ Lincoln County Hospital, UK; ${ }^{8}$ Kettering General Hospital, UK; ${ }^{9}$ Takeda UK Ltd, High Wycombe, UK
\end{abstract}

\subsection{6/gutjnl-2019-BSGAbstracts.193}

Introduction Randomised controlled trials have demonstrated efficacy of vedolizumab in Ulcerative Colitis (UC) and Crohn's Disease (CD). Further data in the real-world setting is needed to inform future practice.

Methods A multicentre retrospective observational chart review study was conducted with all pts initiated on vedolizumab across 7 UK hospitals between 1/11/14-30/11/16. The Health Research Authority approved the protocol (19/HRA/0008). Clinical disease activity was assessed at baseline, week 14, 30 \& 52 using the Harvey Bradshaw Index (HBI) and partial Mayo Score (pMS). Clinical remission was defined as $\mathrm{HBI} \leq 4$ or pMS $<2$ with a combined stool frequency and rectal bleeding subscore of $\leq 1$. Clinical response was defined as $\geq 2$-point decrease from baseline in $\mathrm{pMS}$ and $\geq 3$-point decrease from baseline in HBI. The primary objective was to describe corticosteroid-free and clinical remission. Secondary objectives included effect on disease activity scores, biochemical markers, concomitant drug use, mucosal healing, hospital admissions and adverse effects.

Results 192 patients were included: $100 \mathrm{CD}, 87 \mathrm{UC}$ and 5 IBD unclassified (grouped with CD in this analysis). $46 \%$ of UC and $10 \%$ of CD patients were anti-TNF naïve. Median age was 44 (range 18-79) years; 49\% male and median BMI was 25.7 (IQR 22.5-31.4). Exposure time was 38.0 (23.756.7) for UC and 30.9 (21.3-49.6) weeks for CD. Corticosteroid-free remission rates for UC and CD were $46 \%$ and $45 \%$, while clinical remission rates were $52 \%$ and $44 \%$ respectively. Clinical response rates for UC was $49 \%$ and CD was $53 \%$. The median time to corticosteroid free remission for UC and CD was 17.6 (8.7-29.6) and $14.1(6.0-21.7)$ weeks and clinical remission was $15.1(7.4-24.9)$ and 10.1 (3.1-21.0) weeks respectively. Time to clinical response for UC was 9.4 (5.715.4) and $C D$ was $9.5(6.1-18.2)$ weeks. Disease activity decreased from baseline at 14 weeks: pMS 5 (4-6) vs 3 (1-5) $\mathrm{p}=0.025$ and 30 weeks pMS $5(4-6)$ vs $2(1-5.5) \mathrm{p}=0.032$. Concomitant corticosteroid and immunomodulator use decreased in UC (48\% vs $15 \%$ and $41 \%$ vs $18 \%)$ and CD ( $27 \%$ vs $10 \%$ and $26 \%$ vs $7 \%$ ), respectively. The overall rate of IBD-related hospital admissions per patient per year was 1.3 (0-18.1). Adverse events were reported in $5.2 \%$ of patients.
Conclusions Results in our predominately anti-TNF experienced vedolizumab cohort mirror other published real-world data and demonstrate good clinical effectiveness and safety profile.

\section{PTH-135 AN INFLAMMATORY BOWEL DISEASE-SPECIFIC NUTRITION SCREENING TOOL (IBD-NST) FOR BETTER OUTPATIENT CARE}

${ }^{1}$ Catherine Wall, ${ }^{1,2}{ }^{2}$ Bridgette Wilson*, ${ }^{3}$ Jeremy Sanderson, ${ }^{1,2}$ Miranda Lomer. 'King's College London, Department of Nutritional Sciences, London, UK; ${ }^{2}$ Guy's and St Thomas' NHS Foundation Trust, London, UK; ${ }^{3}$ Guy's and St Thomas' NHS Foundation Trust, Department of Gastroenterology, London, UK

\subsection{6/gutjnl-2019-BSGAbstracts. 194}

Background Patients with inflammatory bowel disease (IBD) can have hidden nutrition risks that generic malnutrition screening tools, which place too much emphasis on body mass index (BMI), are not able to identify, therefore some IBD patients that would benefit from dietary interventions are missed. By identifying nutrition risk using an IBD-specific screening tool, early detection of nutrition risk may be able to prevent development of malnutrition and improve clinical outcomes.

Aim This study aimed to develop and test an IBD-specific nutrition self-screening tool (IBD-NST).

Methods IBD patients were recruited prospectively to independently complete IBD-NST and Malnutrition Universal Screening Tool (MUST). Subjective global assessment (SGA) and hand grip strength (HGS) were completed by a dietitian. The IBD-NST scored nutrition risk as low (0), moderate (1) or high $(\geq 2)$, including questions on BMI, unintentional weight loss, and a combination of active disease and nutrition concerns. Scores were compared to sub-optimal (15\% below the mean) HGS, mid-upper arm muscle circumference (MAMC), BMI, weight loss $>5 \%$ and SGA. Chi ${ }^{2}$ compared dichotomous outcomes and Receiver Operator Characteristic curves were used for prediction assessment with a cut off of AUC $<0.7$ for poor prediction.

\begin{tabular}{|c|c|c|c|c|c|}
\hline & & \multicolumn{4}{|c|}{ MUST } \\
\hline & & Low & Moderate & High & Total \\
\hline \multirow[t]{4}{*}{ IBD-NST } & Low & 60 & 4 & 4 & $68(67 \%)$ \\
\hline & Moderate & 0 & 9 & 0 & $9(9 \%)$ \\
\hline & High & 12 & 1 & 11 & $24(24 \%)$ \\
\hline & Total & $72(71 \%)$ & $14(14 \%)$ & $15(15 \%)$ & 101 \\
\hline
\end{tabular}

Results 101/116 patients (87\%) were recruited, 54 (53\%) were female, 61 (60\%) had CD, 33 (33\%) had UC and 7 (7\%) had IBD-U. Mean (SD) age was 40 (14) years and BMI was $24.6 \mathrm{~kg} / \mathrm{m}^{2}$ (4.3). SGA identified 11/91 (12\%) with malnutrition and IBD-NST and MUST identified a similar number of patients at nutrition risk (table 1). Twelve patients were low risk for MUST but high risk for IBD-NST due to having a flare and concerns about their nutrition. Unlike SGA and MUST, IBD-NST nutrition risk was not predicted by BMI $(\mathrm{AUC}=0.286 \quad(\mathrm{SE} 0.06) \quad(95 \%$ CI $0.17,0.40))$. No significant difference in suboptimal HGS was seen across BMI categories 